\title{
The Right Liver Lobe Size/Albumin Concentration Ratio in Identifying Esophageal Varices among Patients with Liver Cirrhosis
}

\author{
Muhammad Akram ${ }^{1}$, Munawar Hussain Soomro ${ }^{2,3,{ }^{*},}$, Mansoor Magsi ${ }^{4}$
}

1. Department of Internal Medicine, Pakistan Atomic Energy Commission General Hospital, Sector H $-11 / 4$ Islamabad, Pakistan

2. Sorbonne Université and INSERM, Pierre Louis Institute of Epidemiology and Public Health (IPLESP UMRS 1136), Epidemiology of Allergic and Respiratory Diseases Department (EPAR), Faculty of Medicine, SaintAntoine Medical School, Paris, France

3. Department of Community Medicine, Al-Nafees Medical College, Isra University, Islamabad Campus, Islamabad, Pakistan

4. Department of Medicine, Shaheed Mohtarma Benazir Bhutto Medical University, Larkana, Pakistan

\section{* Corresponding Author:}

Munawar Hussain Soomro, MBBS, MSc, MPhil

Sorbonne Université and INSERM, Pierre Louis Institute of Epidemiology and Public Health (IPLESP UMRS 1136), Epidemiology of Allergic and Respiratory Diseases Department (EPAR), Saint-Antoine Medical School, 75012 Paris, France

Tel: +33144738657

Fax: + 33144738454

Email: munawar.soomro@iplesp.upmc.fr

Received: 05 Mar. 2018

Accepted: 20 Jul. 2018

\section{ABSTRACT}

\section{BACKGROUND}

It is necessity to work more on non-invasive markers like right liver lobe size/albumin concentration ratio for predicting esophageal varices. We aimed to see the right liver lobe/albumin ratio in identifying esophageal varices among patients with cirrhosis caused by hepatitis $\mathrm{B}$ and $\mathrm{C}$.

\section{METHODS}

A cross-sectional study was conducted among all indoor patients admitted to the Department of Internal Medicine, Pakistan Atomic Energy Commission, General Hospital, Sector H-11/4 Islamabad, Pakistan, during May-December 2016. A non-probability purposive sampling technique was used for data collection. Diagnosed cases of cirrhosis caused by hepatitis B and C virus infection aged 25 years or more were included. A pre-designed questionnaire was used for data collection. Statistical analysis was done using SPSS software version 20.

\section{RESULTS}

A total number of 160 patients were included. Mean \pm SD age was $56.04 \pm 10.22$ years, while $96(60 \%)$ were men. Child-Turcotte-Pugh grades showed $41.88 \%$ of the patients with grade A, followed by grade B (38.12\%), and grade C (20\%). Esophageal varices were observed in $76.25 \%$ of the patients. The sensitivity was $86.89 \%$, while specificity was $78.95 \%$. The positive predictive value was $92.98 \%(95 \%$ confidence interval $[\mathrm{CI}]=86.64 \%-96.91 \%)$ and negative predictive value was $65.22 \%(\mathrm{CI}=49.75 \%-78.64 \%)$ with a diagnostic accuracy of $85 \%$.

\section{CONCLUSION}

The use of the studied non-invasive markers of portal hypertension particularly the right liver lobe size/albumin concentration ratio, can help physicians to restrict the use of endoscopic screening only to patients presenting a high probability of esophageal varices.

\section{KEYWORDS:}

Portal hypertension, Liver cirrhosis, Esophageal varices, Serum albumin

\section{Please cite this paper as:}

Akram M, Soomro MH, Magsi M. The Right Liver Lobe Size/Albumin Concentration Ratio in Identifying Esophageal Varices among Patients with Liver Cirrhosis. Middle East J Dig Dis 2019;11:32-37. doi: 10.15171/mejdd.2018.125.

\section{INTRODUCTION}

Liver cirrhosis has emerged as one of the major causes of global health burden. ${ }^{1}$ Hepatocellular injury leads to liver cirrhosis due to fibrosis and nodular regeneration throughout the liver. $^{2}$ It is an irreversible stage of liver disease and can be classified as macro- or micro-nodular on the basis of histopathology but the etiological classification is more relevant in clinical scenario. In the order of prevalence, viral hepatitis B and C, and alcoholic liver disease are the 
most common causes followed by autoimmune hepatitis, sclerosing cholangitis, primary biliary cirrhosis, hemochromatosis, and Wilson's disease. ${ }^{1}$ In the West, alcohol appears to be the most common cause of liver cirrhosis, whereas in the developing world viral hepatitis $\mathrm{B}$ and $\mathrm{C}$ is the leading cause. ${ }^{3}$ Hepatitis $\mathrm{C}$ virus is the predominant cause of liver cirrhosis worldwide. ${ }^{4}$

According to the latest report by World Health Organization (WHO), an estimated 257 million people are living with Hepatitis B, while 71 million people have chronic hepatitis $\mathrm{C}$ infection. ${ }^{5}$ Both hepatitis $\mathrm{B}$ and $\mathrm{C}$ cause $8,87,000$ and 3,99,000 deaths each year, respectively worldwide. According to WHO the prevalence of hepatitis $\mathrm{B}$ is the highest in the Western Pacific region $(6.2 \%)$ and the African region (6.1\%), among infected adult population..$^{5}$ In the Eastern Mediterranean region, South-East Asia, and the European region, an estimated $3.3 \%, 2.0 \%$, and $1.6 \%$ of the general population are infected, respectively. While the American region is only infected by $0.7 \%$ of the population, the most affected regions for hepatitis $\mathrm{C}$ are Eastern Mediterranean and European regions, with the prevalence of $2.3 \%$ and $1.5 \%$, respectively. Pakistan ranked in the intermediate prevalence zone for hepatitis $\mathrm{B}$ and $\mathrm{C}$. The overall prevalence of hepatitis $\mathrm{B}$ and $\mathrm{C}$ virus is $2.5 \%$ and $4.8 \%$, respectively. ${ }^{6}$

The prevalence of severe fibrosis (cirrhosis) in patients with chronic hepatitis B is $34 \%$. $^{7,8}$ Complications of long standing liver cirrhosis include portal hypertension, ascites, hepato-renal syndrome, hepatic encephalopathy, and hepatocellular carcinoma. Portal hypertension $(>10 \mathrm{~mm}$ $\mathrm{Hg}$ pressure in the portal vein) occur due to increased resistance of blood flow in the portal vessels and leads to formation of collaterals at the sites of porto-systemic junctions i.e. gastro-esophageal junction, hemorrhoids, and abdominal wall. ${ }^{9,10}$ As a consequence of rising portal pressure, bleeding may start from any of these sites. Bleeding from varices at gastro-esophageal junction can be very serious and life-threatening complication of portal hypertension. Around 25-35\% of patients develop esophageal varices due to portal hypertension, out of them $80-90 \%$ develop variceal bleeding, associated with very high mortality rate. ${ }^{11}$ Initial bleeding episodes are fatal and have been observed in up to $30 \%$ of the patients. ${ }^{11}$ It has been observed that esophageal varices is developed in most of the patients with cirrhosis, with a lifetime incidence as high as $90 \%$. On the other hand, approximately one-third of cirrhotic patients with esophageal varices develop esophageal hemorrhage, which leads to high morbidity and mortality. ${ }^{12}$

Non-invasive indicators of varices are required to reduce the need for screening endoscopy in all patients with cirrhosis. Serum albumin levels are often reduced in patients with liver disease because of a change in the volume of distribution of albumin as well as a reduction in synthesis. Since the plasma half-life of albumin is about three weeks, serum albumin levels may be normal in acute liver failure but are almost always reduced in chronic liver failure. ${ }^{13,14}$

The purpose of this study was to see the right liver lobe size/albumin concentration ratio in identifying esophageal varices among patients with cirrhosis caused by hepatitis $\mathrm{B}$ and $\mathrm{C}$.

\section{MATERIALS AND METHODS}

A cross-sectional study was conducted at the Department of Internal Medicine, Pakistan Atomic Energy Commission (PAEC), General Hospital, Sector H 11/4 Islamabad, Pakistan, during May-December 2016. A non-probability purposive sampling technique was used for data collection. Diagnosed cases of cirrhosis caused by $\mathrm{HBV}$ and $\mathrm{HCV}$ infection (HBs Ag positive, anti-HCV antibodies) confirmed by Enzyme-linked immunosorbent assay (ELIZA) method, aged 25 years or more were included.

All patients with liver cirrhosis and history of alcohol intake, upper gastrointestinal bleeding, history of sclerotherapy/band ligation, receiving prophylactic treatment for portal hypertension, taking diuretics, interferon therapy in the last six months, portal vein thrombosis on ultrasound, or hepatoma on ultrasound were excluded.

\section{Data Collection and Procedure:}

Data were collected from all indoor patients admitted to PAEC Hospital, Islamabad with definite history of liver cirrhosis caused by hepatitis B and $\mathrm{C}$ infection. All patients fulfilled inclusion and exclusion criteria were enrolled in this study. The ethical approval was taken from the Ethical Review Committee before conducting the study. Written informed consent was taken from all 
Table 1: Demographic characteristics of the study population

\begin{tabular}{llcc}
\hline Characteristics & & No & $\mathbf{\%}$ \\
\hline \multirow{2}{*}{ Age } & 25-40 years & 17 & 10.62 \\
\cline { 2 - 4 } & 41-59 years & 69 & 43.13 \\
\cline { 2 - 4 } & 60 years and above & 74 & 46.25 \\
\hline \multirow{2}{*}{ Sex } & Male & 95 & 65.37 \\
\cline { 2 - 4 } & Female & 65 & 40.3 \\
\hline \multirow{2}{*}{$\begin{array}{l}\text { Esophageal } \\
\text { varices }\end{array}$} & Yes & 122 & 76.25 \\
\cline { 2 - 4 } & No & 38 & 23.75 \\
\hline \multirow{2}{*}{$\begin{array}{l}\text { esades of } \\
\text { varices }\end{array}$} & Grade 0 (No varices) & 38 & 23.75 \\
\cline { 2 - 4 } & Grade I & 24.37 \\
\cline { 2 - 4 } & Grade II & 31 & 31.88 \\
\cline { 2 - 4 } & Grade III & 08 & 5.00 \\
\cline { 2 - 4 } & Grade IV & & \\
\hline
\end{tabular}

patients prior to inclusion in the study. General and clinical examination parameters were collected. Further routine laboratory investigations including complete blood tests, Hbs Ag, anti-HCV antibodies by ELIZA method, liver function tests i.e. serum bilirubin, Asparate aminotransferase (AST), Alanine aminotransferase (ALT), alkaline phosphatase, and serum albumin), Prothrombin time (PT), and International normalized ratio (INR) were done. Abdominal ultra-sonographic evaluation for right liver lobe size was performed by a consultant sonologist.

\section{Measurement of right liver lobe diameter:}

While patient lying in a supine position, a curvilinear probe of a high resolution real-time scanner was placed sub-costally in the right mid-clavicular line in a sagittal plane showing a good liver window. The cranio-caudal right lobe diameter at mid-clavicular line was measured from the diaphragm to the liver edge. During the deep inspiration by the patients, measurements were taken to reduce masking by the lung, while the right arm was placed above the head to access the right upper quadrant and to raise the lower costal margin. ${ }^{15,16}$

Patients' right liver lobe size/albumin concentration ratio was calculated by taking 4.42 as cut-off value. Upper gastro-intestinal (GI) endoscopy was performed for evaluation of the presence and grades of esophageal varices. Child-Turcotte-Pugh criteria were used to classify in grades A, B, and C. A pre-designed questionnaire was used for data collection. The effect modifiers were controlled initially by selection criteria (inclusion and exclusion).

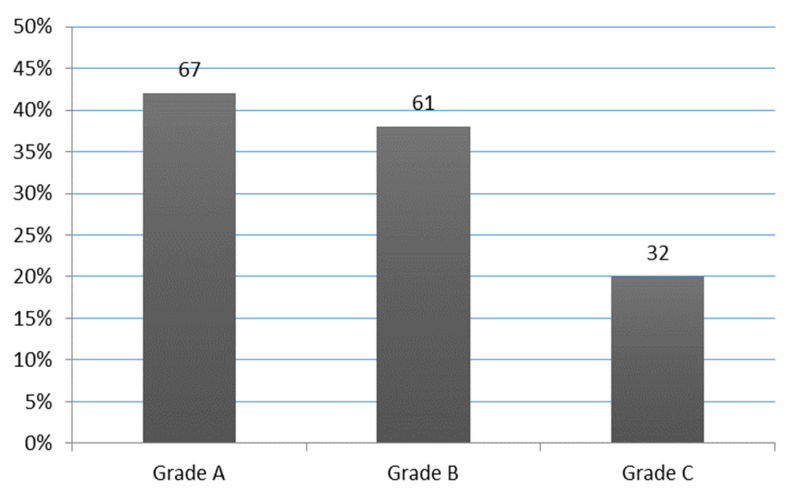

Fig.1: Distribution of Child-Turcotte-Pugh grades

The mean \pm standard deviation (SD) of the variables such as age, right liver lobe size/albumin concentration ratio was calculated. Categorical response variables such as sex $(\mathrm{M} / \mathrm{F})$, anti-HCV, HBs Ag, and Child-Pugh class were presented in frequency and proportion. Sensitivity, specificity, positive predictive value, negative predictive value, and confidence interval were calculated. A receiver operator characteristic (ROC) analysis was used to determine the optimum cut-off value for the diagnostic marker. All the statistical analysis was conducted using SPSS software version 20. $P$ value $<0.05$ was considered as statistically significant.

\section{RESULTS}

In this study 160 patients were included. The demographic characteristics of the study population are shown in table 1 . Mean $\pm \mathrm{SD}$ age was $56.04 \pm 10.22$ years. There were $96(60 \%)$ men and almost half $(46.25 \%)$ of the study population was among older age group. The distribution of Child-Turcotte-Pugh grades shows that 67 (41.88\%) patients had grade A, followed by 61 (38.12\%) patients with grade B and 32 (20\%) patients with grade $\mathrm{C}$ as shown in figure 1 . The mean $\pm \mathrm{SD}$ values of right liver lobe size were $130.24 \pm 5.68 \mathrm{~cm}^{2}$ (range: $113-142$ $\mathrm{cm}^{2}$ ). The mean \pm SD serum albumen level was $23.58 \pm$ $3.72 \mathrm{~g} / \mathrm{dL}$, having a minimum value of 15 and maximum value of $33 \mathrm{~g} / \mathrm{dL}$. The mean \pm SD liver lobe/albumin ratio were $5.53 \pm 0.94$. The minimum and maximum values of right liver lobe size/albumin concentration ratio were 4.20 and 7.87 , respectively. 
Table 2: The right liver lobe size/albumin concentration ratio with respect to upper gastrointestinal endoscopy grades

\begin{tabular}{lccccc}
\hline $\begin{array}{l}\text { Upper gastrointestinal } \\
\text { endoscopy (esophageal varices) }\end{array}$ & $\mathbf{n}(\%)$ & Minimum & Maximum & Mean & Std. deviation \\
\hline No varices (grade 0) & $38(23.75)$ & 4.20 & 4.89 & 4.35 & .15 \\
\hline Grade I & $39(24.37)$ & 4.90 & 6.28 & 5.03 & .61 \\
\hline Grade II & $51(31.88)$ & 5.56 & 6.23 & 6.00 & .12 \\
\hline Grade III & $24(15.00)$ & 6.50 & 6.66 & 6.59 & .04 \\
\hline Grade IV & $8(5.00)$ & 6.77 & 7.87 & 7.22 & .36 \\
\hline
\end{tabular}

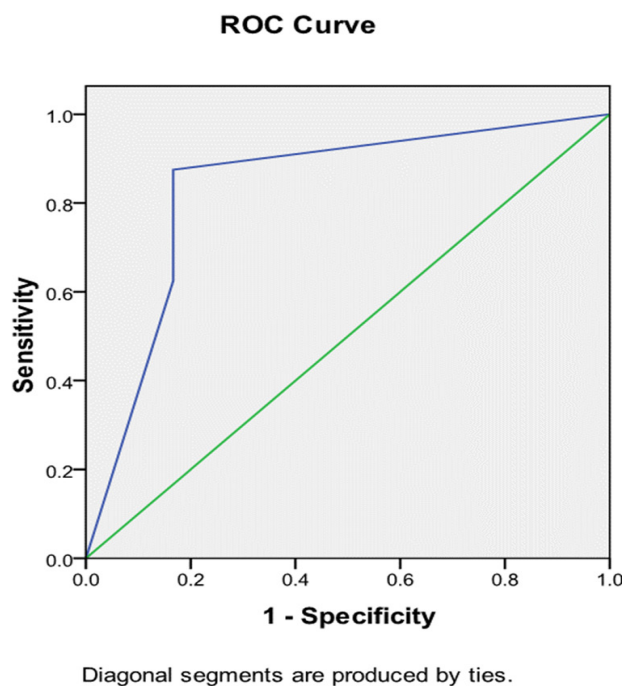

Fig.2: ROC curve for sensitivity and specificity of right liver lobe size/albumin concentration ratio for the prediction of varices

We observed that $51(31.88 \%)$ patients had esophageal varices grade II, followed by 39 (24.37\%) patients with grad I esophageal varices. Grade III and grade IV esophageal varices were found in $24(15.00 \%)$ and $8(5.00 \%)$ patients, respectively. There was grade 0 or no varices in $38(23.75 \%)$ patients as shown in table 2 . The mean $\pm \mathrm{SD}$ right liver lobe size/albumin concentration ratio in grade 0 patients was $4.35 \pm 0.15$. The mean $\pm \mathrm{SD}$ values of right liver lobe size/albumin concentration ratio in grade I, grade II, grade III, and grade IV esophageal varices by upper GI endoscopy were $5.03 \pm 0.61,6.01 \pm 0.12,6.59 \pm$ 0.04 , and $7.22 \pm 0.36$, respectively. The overall distribution of esophageal varices assessed by endoscopy shows that there were $122(76.25 \%)$ positive cases.

By applying ROC curves, the sensitivity, specificity, and accuracy of the right liver lobe diameter/albumin concentration ratio as non-invasive predictors of the presence of esophageal varices and large varices were studied (figure 2). In our study the sensitivity was $86.89 \%$ (95\% confidence interval $[\mathrm{CI}]=79.58-92.31$ ) for right liver lobe size/albumin concentration ratio. The specificity was $78.95 \%(\mathrm{CI}=62.67-90.42)$, positive predictive value was $92.98 \%(\mathrm{CI}=86.64-96.91)$ and negative predictive value was $65.22 \%(\mathrm{CI}=49.75-78.64)$. We observed a diagnostic accuracy of $85 \%$ in our study.

\section{DISCUSSION}

The prevention of esophageal varices bleeding is important. The identification of patients at the risk of variceal bleeding is the first step. Non-invasive markers of esophageal varices have attracted the interest of investigators in recent years and studies have shown, which predict the presence of esophageal varices in liver cirrhosis with a goal to reduce the burden and cost on endoscopy. ${ }^{15,17,18}$

In our study there were $122(76.25 \%)$ patients with esophageal varices, similar results were found in studies from Egypt, Serbia, India, and Pakistan. ${ }^{15,19-21}$ Whereas a cross-sectional study from Lahore, Pakistan by Khan and colleagues ${ }^{22}$ showed only a $22.3 \%$ of patients with esophageal varices. Another study conducted on 197 patients from Peshawar showed esophageal varices in $32 \%$ of the patients. ${ }^{23}$ There might be a reason that about $46 \%$ of the population in our study was among old age group. In our study, a cut-off value of 4.42 was taken, while according to the Child-Turcotte-Pugh grades we observed 67 (41.88\%) patients with grade A, followed by $61(38.12 \%)$ patients with grade B, and $32(20 \%)$ patients with grade $\mathrm{C}$, which is consistent with the findings reported in previous studies. ${ }^{19,21} \mathrm{~A}$ Serbian study by Alempijevic et.al. ${ }^{19}$ showed that $24.5 \%$ of the patients with no esophageal varices were identified by upper GI endoscopy, 22.3\% had grade I esophageal varices, $33 \%$ had grade II esophageal varices, $16 \%$ had grade III esophageal varices, and $4.3 \%$ had grade IV esophageal 
varices. A cut-off value of 4.425 was taken and the mean value of the right liver lobe diameter/albumin concentration ratio was $5.51 \pm 1.82$ (range: 2.76 - 11.44). The sensitivity was $83.1 \%$ and the specificity was $73.9 \%$.

Other published studies have also reported that platelet count/spleen size ratios are good predictors of esophageal varices. ${ }^{17,24-27}$ Despite a good correlation between these ratios and esophageal varices grade, it is unlikely that these ratios could be used to exclude patients from initial endoscopic screening. Nevertheless, these ratios may serve for selection of patients who need more frequent endoscopies. These ratios will help to identify patients at higher risks for development of esophageal varices. ${ }^{19}$ Endoscopy is still the gold standard method for diagnosis of esophageal varices and is recommended that cirrhotic patients without varices receive an examination every two to three years and that patients with small varices receive an examination every one to two years. ${ }^{17,28} \mathrm{Al}-$ though the results of the non-invasive predictor analysis, the right liver lobe diameter/albumin concentration ratio, appear to be promising, yet cannot replace endoscopy.

Our study has strengths as well as some limitations. Cross-sectional studies are useful for description, and we chose this design for our study. The primary investigator collected all the data on a pre-designed form. The study was conducted in one district of the country and did not cover other districts and the situation in other districts can be different. Other limitations include the absence of a comparative group, the small sample size, and the limited time of this study.

Although our results may not be generalized to the entire Pakistani population, however national and international studies have shown similar results as ours. Further studies are necessary with large sample size.

\section{CONCLUSION}

The use of the studied non-invasive marker of portal hypertension, the right liver lobe size/albumin concentration ratio, can help physicians to restrict the use of endoscopic screening only to patients presenting a high probability of esophageal varices.

\section{Authors' Contributions:}

MA participated in study design, data collection, data entry and analysis, data interpretation, draft writing and editing. MM participated in study design, analysis, data interpretation, draft writing and editing. MHS participated in study design, data analysis and interpretation, draft writing, editing and submission of the manuscript. All authors read and approved the final manuscript.

\section{ACKNOWLEDGMENTS}

Our deepest appreciations to all the study participants.

\section{Data Confidentiality:}

Confidentiality was ensured by not disclosing the identity of participants.

\section{Funding Sources:}

None

\section{ETHICAL APPROVAL}

There is nothing to be declared.

\section{CONFLICT OF INTEREST}

The authors declare no conflict of interest related to this work.

\section{REFERENCES}

1. Mokdad AA, Lopez AD, Shahraz S, Lozano R, Mokdad AH, Stanaway J, et al. Liver cirrhosis mortality in 187 countries between 1980 and 2010: a systematic analysis. BMC Med 2014;12:145. doi:10.1186/s12916-014-0145-y.

2. Schuppan D, Afdhal NH. Liver Cirrhosis. Lancet 2008:371:838-51. doi:10.1016/S0140-6736(08)60383-9.

3. Pinter M, Trauner M, Peck-Radosavljevic M, Sieghart W. Cancer and liver cirrhosis: implications on prognosis and management. ESMO Open 2016;1:e000042. doi: 10.1136/esmoopen-2016-000042.

4. Ke PY, Chen SS. Autophagy in hepatitis C virus-host interactions: Potential roles and therapeutic targets for liver-associated diseases. World $J$ Gastroenterol 2014;20:5773-93. doi:10.3748/wjg.v20.i19.5773.

5. World Health Organization. Global Hepatitis Report 2017. Geneva: ISBN 978-92-4-156545-5. 2017.

6. Butt AS. Epidemiology of Viral Hepatitis and Liver Diseases in Pakistan. Euroasian J Hepatogastroenterol 2015;5:43-8. doi:10.5005/jp-journals-10018-1129.

7. Fung J, Lai CL, But D, Wong D, Cheung TK, Yuen MF. Prevalence of fibrosis and cirrhosis in chronic hepatitis B: implications for treatment and management. Am J Gastroenterol 2008;103:1421-6. doi:10.1111/j.1572-0241.2007.01751.x.

8. Göbel T, Erhardt A, Herwig M, Poremba C, Baldus SE, Sagir A, et al. High prevalence of significant liver fibro- 
sis and cirrhosis in chronic hepatitis B patients with normal ALT in central Europe. J Med Virol 2011;83:968-73. doi:10.1002/jmv.22048.

9. Sansoè G, Aragno M, Mastrocola R, Mengozzi G, Novo E, Parola M. Role of Chymase in the Development of Liver Cirrhosis and Its Complications: Experimental and Human Data. PLoS One 2016;11: e0162644. doi:10.1371/ journal.pone.0162644.

10. Nusrat S, Khan MS, Fazili J, Madhoun MF. Cirrhosis and its complications: Evidence based treatment. World $J$ Gastroenterol 2014;20:5442-60. doi:10.3748/wjg.v20. i18.5442.

11. Sharara AI, Rockey DC. Gastroesophageal variceal hemorrhage. N Engl J Med 2001;345:669-81. doi:10.1056/ NEJMra003007.

12. Chang YW. Indication of treatment for esophageal varices: who and when? Dig Endosc 2006;18:10-15. doi:10.1111/j.1443-1661.2006.00579.x.

13. Liumbruno G, Bennardello F, Lattanzio A, Piccoli P, Rossettias G, Italian Society of Transfusion Medicine and Immunohaematology (SIMTI). Recommendations for the use of albumin and immunoglobulins. Blood Transfus 2009;7:216-34. doi:10.2450/2009.0094-09.

14. Andersen JT, Dalhus B, Viuff D, Ravn BT, Gunnarsen KS, Plumridge A, et al. Extending Serum Half-life of Albumin by Engineering Neonatal Fc Receptor (FcRn) Binding. J Biol Chem 2014;289:13492-502. doi:10.1074/ jbc.M114.549832.

15. Esmat S, Omarn D, Rashid L. Can we consider the right hepatic lobe size/albumin ratio a noninvasive predictor of oesophageal varices in hepatitis C virus-related liver cirrhotic Egyptian patients? Eur J Intern Med 2012;23:26772. doi:10.1016/j.ejim.2011.11.010.

16. Riestra-CandelariaBL, Rodriguez-MojicaW, Vazquez-Quinones LE, Jorge JC. Ultrasound Accuracy of Liver Length Measurement with Cadaveric Specimens. J Diagn Med Sonogr 2016;32:12-9. doi:10.1177/8756479315621287.

17. Giannini E, Botta F, Borro P, Risso D, Romagnoli P, Fasoli A, et al. Platelet count/spleen diameter ratio: proposal and validation of a non-invasive parameter to predict the presence of oesophageal varices in patients with liver cirrhosis. Gut 2003;52:1200-5.

18. Frank C, Mohamed MK, Strickland GT, Lavanchy D, Arthur RR, Magder LS, et al. The role of parenteral antischistosomal therapy in the spread of hepatitis $\mathrm{C}$ virus in Egypt. Lancet 2000;355:887-91. doi:10.1016/S01406736(99)06527-7.

19. Alempijevic T, Bulat V, Djuranovic S, Kovacevic N, Jesic R, Tomic D, et al. Right liver lobe/albumin ratio: Contribution to non-invasive assessment of portal hypertension. World J Gastroenterol 2007;13:5331-5.

20. Chandail VS, Kotwal SK, Koul S, Gupta R, Mahajan A. Non-invasive markers for prediction of varices in patients with portal hypertension. Int J Res Med Sci 2017;5:100710. doi:10.18203/2320-6012.ijrms20170652.
21. Mandhwani R, Hanif FM, Haque MMU, Wadhwa RK, Luck NH, Mubarak M. Noninvasive clinical predictors of portal hypertensive gastropathy in patients with liver cirrhosis. J Transl Intern Med 2017;5:169-73. doi:10.1515/ jtim-2017-0025.

22. Khan J, Rabbani A, Ali S, et al. Frequency of Esophageal Varices and Comparison of Serum Albumin levels with and without Esophageal Varices in Patients Presenting with Chronic Liver Disease. PJMHS 2016;10:127-9.

23. Khan H, Iman N. Hypoalbuminemia: a marker of esophageal varices in chronic liver disease due to hepatitis B and C. Rawal Med J 2009;34:98-101.

24. Baig WW, Nagaraja MV, Varma M, Prabhu R. Platelet count to spleen diameter ratio for the diagnosis of esophageal varices: Is it feasible? Can J Gastroenterol 2008;22:825-8. doi:10.1155/2008/287274.

25. Thabut D, Ratziu V, Trabut JB, Poynard T. Prediction of oesophageal varices with platelet count/spleen diameter ratio or platelets alone. Gut 2004;53:913-4.

26. Gonzalez-Ojeda A, Cervantes-Guevara G, Chavez-Sanchez M, Dávalos-Cobián C, Ornelas-Cázares S, MacíasAmezcua MD, et al. Platelet count/spleen diameter ratio to predict esophageal varices in Mexican patients with hepatic cirrhosis. World J Gastroenterol 2014;20:207984. doi:10.3748/wjg.v20.i8.2079.

27. Colli A, Gana JC, Yap J, Adams-Webber T, Rashkovan $\mathrm{N}$, Ling SC, et al. Platelet count, spleen length, and platelet count-to-spleen length ratio for the diagnosis of oesophageal varices in people with chronic liver disease or portal vein thrombosis. Cochrane Database Syst Rev 2017;4:CD008759.

28. Moctezuma-Velazquez C, Abraldes JG. Non-invasive diagnosis of esophageal varices after Baveno VI. Turk J Gastroenterol 2017;28:159-65. doi:10.5152/tjg.2017.16744. 